\title{
Laser-induced rotation of a levitated sample in vacuum
}

\author{
Won-Kyu Rhim ${ }^{\text {a) }}$ and Paul-François Paradis \\ Jet Propulsion Laboratory, California Institute of Technology, Pasadena, California 91109
}

(Received 3 May 1999; accepted for publication 15 September 1999)

\begin{abstract}
A method of systematically controlling the rotational state of a sample levitated in a high vacuum using the photon pressure is described. A zirconium sphere was levitated in the high-temperature electrostatic levitator and it was rotated by irradiating it with a narrow beam of a high-power laser on a spot off the center of mass. While the laser beam heated the sample, it also rotated the sample with a torque that was proportional both to the laser power and the length of the torque arm. A simple theoretical basis was given and its validity was demonstrated using a solid zirconium sphere at $\sim 2000 \mathrm{~K}$. This method will be useful to systematically control the rotational state of a levitated sample for the containerless materials processing at high temperature. (C) 1999 American Institute of Physics. [S0034-6748(99)04712-7]
\end{abstract}

\section{INTRODUCTION}

A levitated sample in the high-temperature levitator usually ends up in a rotational state. Various thermophysical property measurement techniques assume that the sample is in a nonrotating state for correct measurements. For example, the surface tension and viscosity measurements from a rotating melt will give quite different results than those measured from a static melt. Conventionally, a strong dc magnetic field was applied to the sample to slow down the rotation. A recently developed method utilized the torque generated by an applied rotating magnetic field in order to systematically control the rotational state of the sample. ${ }^{1}$ However, these methods are often quite difficult to implement if the full three-dimensional rotation control is needed. Furthermore, the application of these methods is limited to relatively good electrical conductors. When the sample is an insulator or a poor conductor, these methods no longer apply.

This article describes an alternative method for inducing sample rotation by photon pressure. The technique is capable of systematically altering the rotational state of a levitated sample, either solid or molten, by simply steering a steadystate laser beam at different points on the sample surface. Good electrical conductivity is not required as long the sample material can be levitated in a high vacuum and as long as it can withstand the high temperature resulting from the laser absorption. As far as the conversion of photon pressure to mechanical motion is concerned, there exists the optical levitation technique that was pioneered by Ashkin, ${ }^{2,3}$ and the sample rotation technique by Higurashi et al. who have demonstrated that under an appropriate condition the photon pressure can induce rotations on a small dielectric rotor. ${ }^{4}$ However, their works involve micron-size dielectric particles which are much smaller in size and also restricted to the dielectric materials.

The theoretical justification will be followed by a brief description of the high-temperature, high-vacuum electrostatic levitator used at the Jet Propulsion Laboratory (JPL).

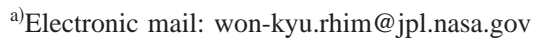

A qualitative verification of the method will be given by showing the rotation that was induced on a levitated molten zirconium drop. A more rigorous experimental verification will then follow.

\section{THEORETICAL ANALYSIS}

If a narrow cw laser beam is pointing a levitated sample (which is opaque at the laser wave length), a part of the laser power will be absorbed and the rest will be reflected. The absorbed laser energy will raise the sample temperature to a steady state. At the steady state in a high vacuum, the total absorbed laser power will be radiated by the sample according to the following equation:

$$
P_{\mathrm{ab}}=N_{\mathrm{ab}} h \nu=\frac{N_{\mathrm{ab}} h c}{\lambda}=\sigma_{\mathrm{SB}} \epsilon_{T} A T^{4},
$$

where $N_{\mathrm{ab}}$ is the number of photons absorbed by the sample per second, $h$ is the Planck constant, $\nu$ is the laser frequency, $c$ is the speed of light, $\lambda$ is the laser wavelength, $\sigma_{\mathrm{SB}}$ is the Stefan-Boltzman constant, $\epsilon_{T}$ is the hemispherical total emissivity, $A$ is the sample surface area, and $T$ is the steadystate sample temperature in kelvin. Since the rest of the total incident laser power must be reflected,

$$
P_{\text {total }}=P_{\mathrm{ab}}+P_{\text {ref }} .
$$

Since a photon with wavelength $\lambda$ has the momentum $p$ $=h / \lambda$, the total momentum that is transferred to the sample by the absorbed photons per second is given by

$$
p_{T}=\frac{N_{\mathrm{ab}} h}{\lambda}=\frac{P_{\mathrm{ab}}}{c} .
$$

If the laser beam incident to the sample surface at the arm length $a$ (see Fig. 1) was absorbed, the torque imparted to the sample by the beam is

$$
\tau=a p_{T}=\frac{a P_{\mathrm{ab}}}{c} .
$$




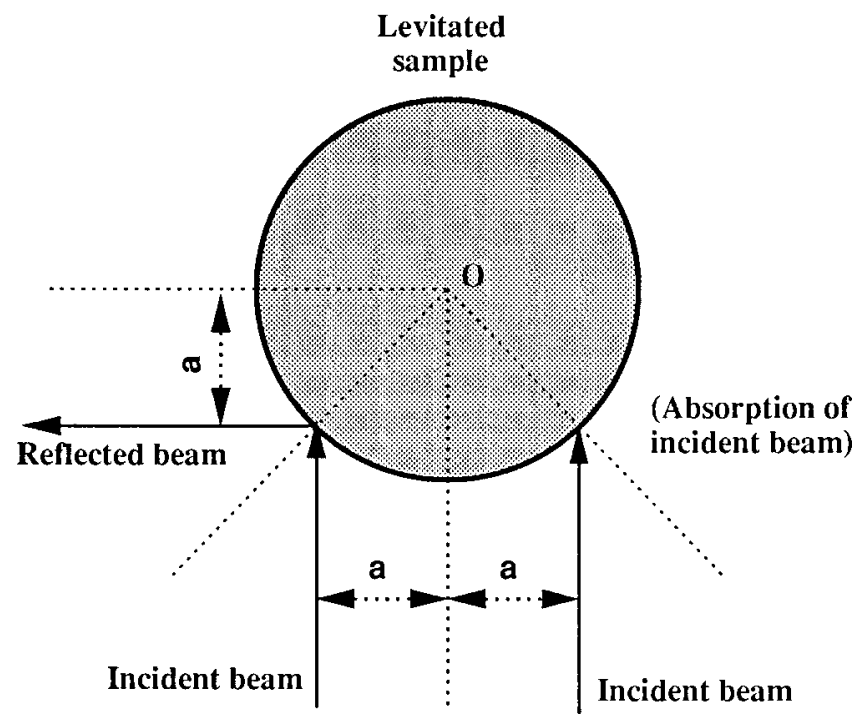

FIG. 1. Schematic diagram showing the two different ways of the laser beams interacting with a spherical sample. One beam is reflected from the surface, while the other beam (incident a distance away from the center) is absorbed.

That is, the torque is proportional to the absorbed laser power and the torque arm length (see Fig. 1). Combination of Eqs. (4) and (1) gives

$$
\tau=\frac{\sigma_{\mathrm{SB}} \epsilon_{T} A a T^{4}}{c}
$$

For a spherical sample of mass $m$, radius $R$, surface area $A=4 \pi R^{2}$, and moment of inertia $I=(2 / 5) m R^{2}$, the acceleration of rotational frequency can be found from Eq. (5) by

$$
\frac{d f}{d t}=\frac{5 \sigma_{\mathrm{SB}}}{c}\left(\frac{\epsilon_{T} a T^{4}}{m}\right),
$$

since torque is defined by

$$
\tau=I \frac{d \omega}{d t}=2 \pi I \frac{d f}{d t},
$$

where $\omega$ is the angular frequency of the sample, and $\omega$ $=2 \pi f$.

The reflected laser beam can also impart a torque to the sample if the sample is nonspherical. The magnitude and direction of the torque generated by the reflected laser beam depend on the details of the area of reflection with respect to the center of sample mass. However, as illustrated in Fig. 1, if the sample shape is spherical having a mirror-smooth surface, the reflected laser beam cannot generate a net torque since the torque generated by the reflected beam cancels the torque generated by the incident beam. Thus, for a spherical sample, the torque has to be generated entirely by the absorbed laser beam as expressed by Eq. (5).

\section{EXPERIMENTAL SETUP AND PROCEDURE}

The experiments reported in this article have been conducted using the high-temperature electrostatic levitator (HTESL) at the Jet Propulsion Laboratory. ${ }^{5}$ Figure 2 shows a schematic diagram of the HTESL. The sample and electrodes were housed in a stainless-steel chamber which was typically

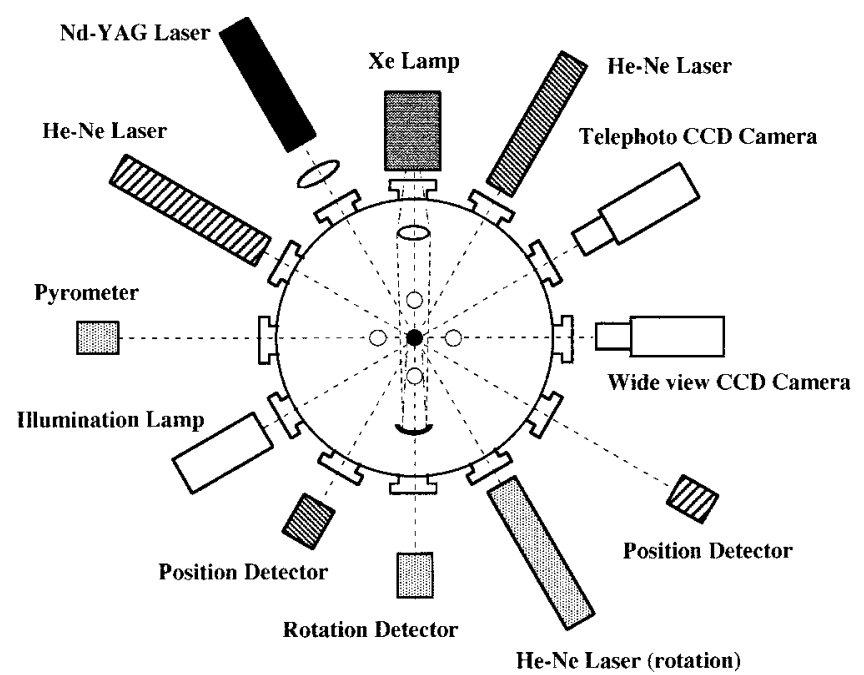

FIG. 2. Schematic diagram of the high-temperature electrostatic levitator.

evacuated to $10^{-8}$ Torr before heating began. For these measurements, samples with diameters close to $2.5 \mathrm{~mm}$ were used. The HTESL achieved sample positioning between the parallel-plate electrodes using a feedback control system. This system relied on $\mathrm{He}-\mathrm{Ne}$ lasers that projected sample images on position detectors. Typically, the top and bottom electrodes were spaced $12 \mathrm{~mm}$ apart (Fig. 3). The sample rotation rate was measured by detecting the reflected $\mathrm{He}-\mathrm{Ne}$ laser beam from its surface. A $1 \mathrm{~kW}$ xenon arc lamp was used for initial sample heating, until a thermionic emission regime was attained. Then, the sample was heated further and melted using a $100 \mathrm{~W} \mathrm{cw} \mathrm{Nd-YAG} \mathrm{laser.} \mathrm{For} \mathrm{these}$ experiments, temperatures in excess of $2300 \mathrm{~K}$ were reached.

When a levitated sample is molten, it assumes a nearly spherical shape, thanks to the action of surface tension. Moreover, since the electrostatic levitation scheme does not transfer any heat to the sample, a heated sample cools purely radiatively. The energy equation describing the cooling process in the absence of the heating beam is given by

$$
\frac{m}{M} C_{p} \frac{d T}{d t}=-\epsilon_{T} A \sigma_{\mathrm{SB}}\left(T^{4}-T_{a}^{4}\right),
$$

where $m$ is the sample mass; $M$ the atomic weight; $C_{p}$ the

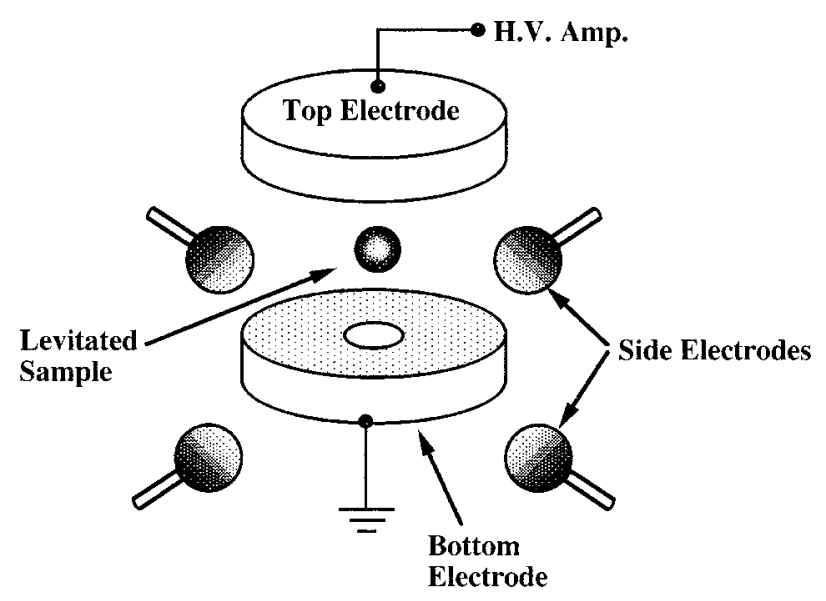

FIG. 3. Schematic diagram of the electrode assembly. 


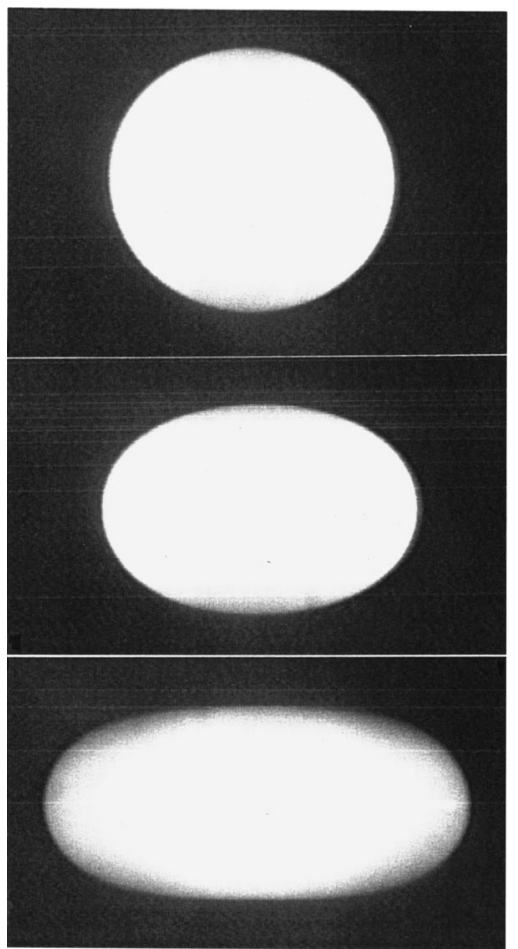

FIG. 4. Side views of a molten zirconium drop rotating at three different rates. The top figure shows the initial spherical shape of the drop in the nonrotating state. The middle figure shows the drop shape near the critical point, and the bottom figure shows a triaxial ellipsoidal shape that occurred past the critical point.

molar heat capacity at constant pressure; $T_{a}$ and $T$ are, respectively, the ambient and sample temperatures; $\epsilon_{T}$ the hemispherical total emissivity; $A$ the surface area of the sample; and $\sigma_{\mathrm{SB}}$ the Stefan-Boltzman constant (5.6705 $\times 10^{-8} \mathrm{~W} \mathrm{~m}^{-2} \mathrm{~K}^{-4}$ ).

The temperature was measured using a single-color custom-made pyrometer operating at $750 \mathrm{~nm}$ and was then digitized and calibrated using LABVIEW software. Since the heating was achieved with a laser at $1.06 \mu \mathrm{m}$, the risks of spectral interference were avoided.

\section{EXPERIMENTAL RESULTS AND DISCUSSIONS}

A solid zirconium sample (melting temperature $2128 \mathrm{~K}$ ) was levitated, and it was heated beyond the melting temperature by the laser. When the laser beam was deliberately pointed to one side of the sample along the equator, the drop started gaining angular momentum, which was evidenced by the shape transformation from spherical to an oblate spheroid. Shown in Fig. 4 are the side views of the molten zirconium drop $(\sim 40 \mathrm{mg})$ at three different rotational states. The top figure shows the initial spherical shape of the drop in the nonrotating state. As the drop continued gaining angular momentum, it was expected to reach the critical point called "the bifurcation point" where the drop shape should suddenly change from an axisymmetric to a triaxial shape. ${ }^{6}$ The middle figure shows the drop shape near the critical point. The bottom figure shows a triaxial ellipsoidal shape that occurred past the critical point. When the laser beam was

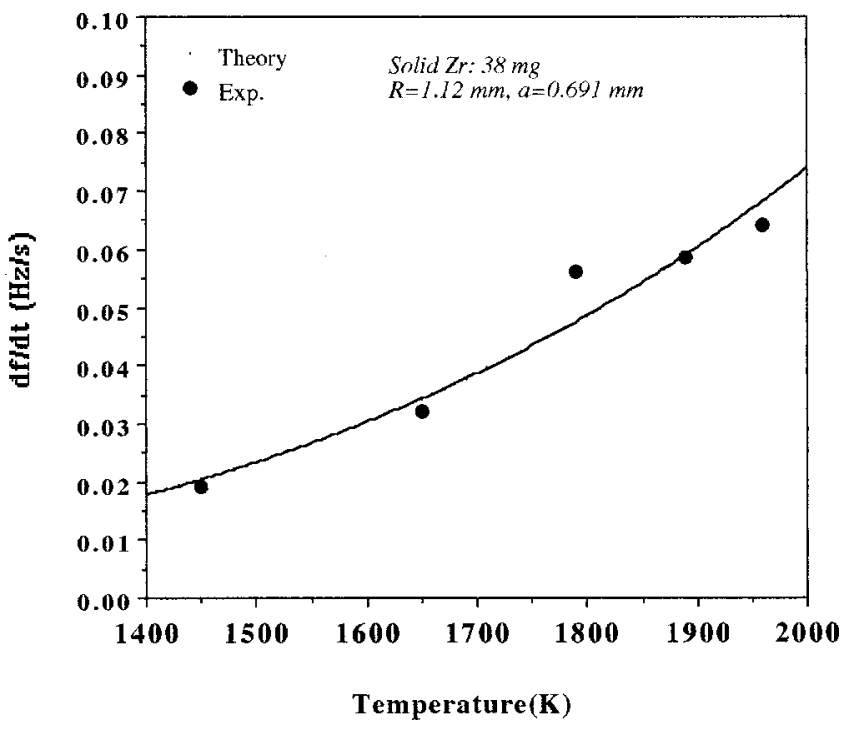

FIG. 5. Theoretical and experimental results of the rotational acceleration of a zirconium sphere as a function of the temperature. The torque arm was kept fixed.

moved to a point that was opposite with respect to the center of the drop, the drop rotational rate decreased as expected.

In order to verify the rotational acceleration given by Eq. (6), a spherical solid zirconium sample was used to maintain the moment of inertia relatively constant throughout the experiment. Sphericity of the sample was ensured by solidifying molten zirconium drop during levitation. The solid sample had the following characteristics at $\sim 2000 \mathrm{~K}$ where the experiment was performed:

mass: $m=3.8 \times 10^{-6} \mathrm{~kg}$,
density: $\rho=6.5 \times 10^{3} \mathrm{~kg} / \mathrm{m}^{3}$,
radius: $R=1.12 \times 10^{-3} \mathrm{~m}$,

moment of inertia: $I=2 m R^{2} / 5=19 \times 10^{-2} \mathrm{~kg} \mathrm{~m}^{2}$,

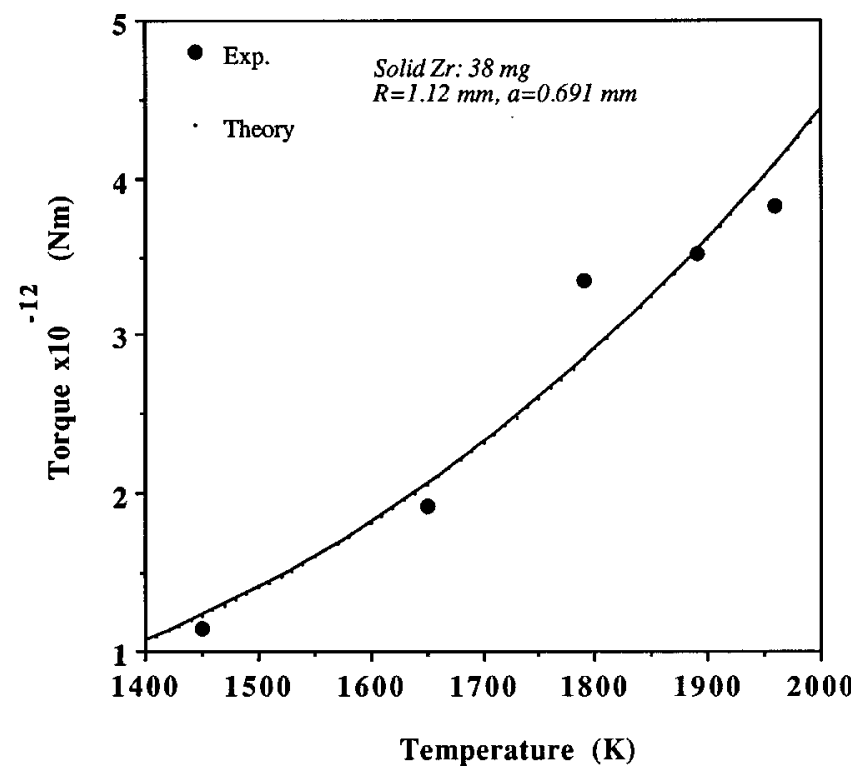

FIG. 6. Theoretical and experimental results of the torque of a zirconium sphere as a function of the temperature. The torque arm was kept fixed. 


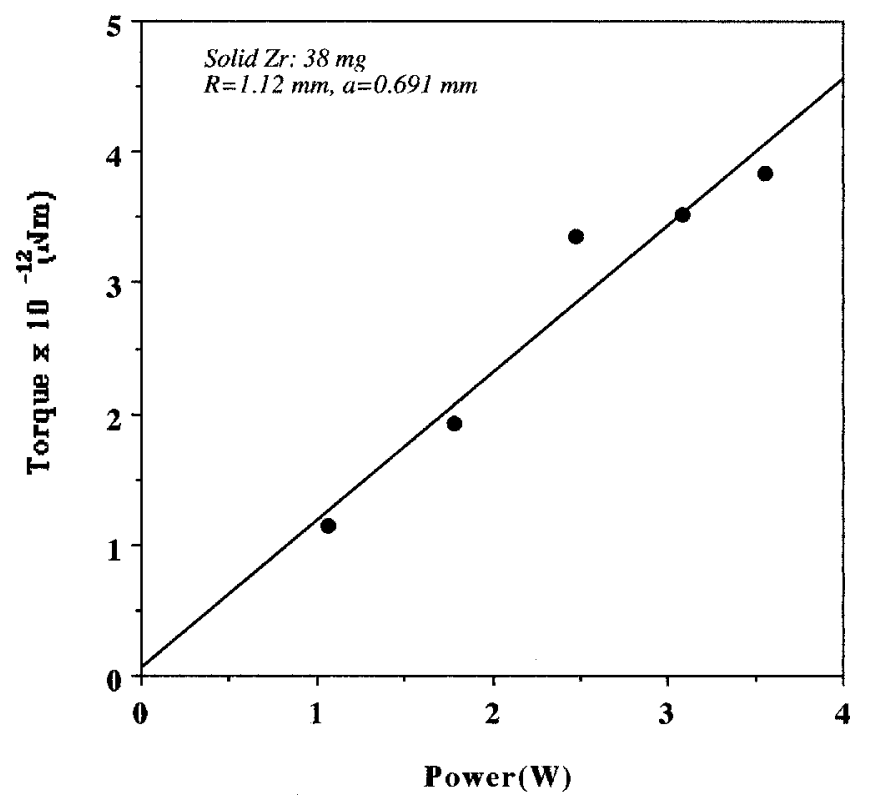

FIG. 7. Theoretical and experimental results of the torque of a zirconium sphere as a function of the absorbed laser power. The torque arm was kept fixed.

hemispherical total emissivity: $\epsilon_{T}=0.27$.

To measure the rotational rate accurately, a low-power $\mathrm{He}-\mathrm{Ne}$ laser was pointed to the sample and the reflected beam was spectrum analyzed. ${ }^{1}$

The laser beam was directed to a fixed point on the sample equator, a short distance away from the center of the sphere. At a given laser power, both the sample rotation rate and the sample temperature were measured. This process was repeated as a function of the laser power. Assuming that the laser beam was pointing $0.69 \mathrm{~mm}$ away from the center of the sample (see Fig. 1), i.e., if the torque arm was 0.69 $\mathrm{mm}$, one can calculate $d f / d t$ from Eq. (6). The results are shown as the solid line in Fig. 5. The experimental results are shown as the solid circles in Fig. 5. The agreements are quite good. In this analysis, the exact torque arm length was the only parameter that needed to be adjusted to fit the data.
Figure 6 shows both the experimental and the theoretical torque, which was translated from Fig. 5 using Eqs. (5) and (7). The torque shown in Fig. 6 can be plotted as a function of the absorbed laser power as shown in Fig. 7. At the torque arm length $a=0.69 \mathrm{~mm}$, the torque generation efficiency by the absorbed laser power was $1.13 \times 10^{-12} \mathrm{~N} \mathrm{~m} / \mathrm{W}$.

This analysis demonstrated that the photon pressure can generate a torque on a levitated sample which can be used to control the sample rotation by simply steering the beam to an appropriate point on the sample surface. If the sample has a spherical shape, only the absorbed laser beam can generate the torque. However, if the sample has a nonspherical shape, both the absorbed and the reflected beams can generate the torque unless the beam is directed to the center of the sample mass. As can be seen in Fig. 5, the sample had to absorb substantial laser power to generate an appreciable rotational rate, meaning that this technique for the rotation control may well be applied to the containerless materials processing at high temperature.

\section{ACKNOWLEDGMENTS}

The authors would like to thank Dr. Eugene Trinh for lending them the $\mathrm{Nd}-\mathrm{YAG}$ laser used in this experiment. The authors also would like to express their thanks to Professor W. Johnson of Caltech who pointed out that the torque generated by the laser beam reflected from a spherical sample negates the torque generated by the incident beam, giving a net zero torque. This work was carried out at the Jet Propulsion Laboratory, California Institute of Technology, under a contract with the National Aeronautics and Space Administration.

${ }^{1}$ W. K. Rhim and T. Ishikawa, Rev. Sci. Instrum. 69, 3628 (1998).

${ }^{2}$ A. Ashkin, Phys. Rev. Lett. 24, 156 (1970).

${ }^{3}$ A. Ashkin, Proc. Natl. Acad. Sci. USA 94, 4853 (1997).

${ }^{4}$ E. Higurashi, H. Ukita, H. Tanaka, and O. Ohguchi, Appl. Phys. Lett. 64, 2209 (1994).

${ }^{5}$ W. K. Rhim, S. K. Chung, D. Barber, K. F. Man, G. Gutt, A. Rulison, and R. E. Spjut, Rev. Sci. Instrum. 64, 2961 (1993).

${ }^{6}$ P. M. Adornado and R. A. Brown, Proc. R. Soc. London, Ser. A 389, 101 (1983). 